\title{
Paraneoplastic Motor Neuron Disease Resembling Amyotrophic Lateral Sclerosis in a Patient with Renal Cell Carcinoma
}

\author{
H. Mehmet Turk ${ }^{a} \quad$ Ahmet Ozet $^{b}$ Okan Kuzhan ${ }^{b}$ Ferhan Komurcu ${ }^{c}$ \\ Fikret Arpaci $^{\mathrm{b}}$ Bekir Ozturk ${ }^{\mathrm{b}}$ Selmin Ataergin ${ }^{\mathrm{b}}$ \\ a Department of Clinical Oncology, Faculty of Medicine, Gaziantep University, Gaziantep, bepartment of Clinical \\ Oncology, GATA Faculty of Medicine, and ' Department of Neurology, Ankara Ataturk Hospital, Ankara, Turkey
}

\section{Key Words}

Amyotrophic lateral sclerosis · Paraneoplastic syndrome •

Renal cell carcinoma

\begin{abstract}
Objective: To report an unusual paraneoplastic syndrome, amyotrophic lateral sclerosis, associated with renal cell carcinoma. Case Presentation and Intervention: A 59-year-old man presented with muscle weakness and fasciculations in the upper extremities. Neurological examination showed that the fasciculations arose spontaneously in the upper limbs. Electrodiagnostic studies revealed an active neurogenic disorder. The patient was diagnosed with a motor neuron disease mimicking amyotrophic lateral sclerosis. Urine analysis revealed microscopic hematuria. Abdominal computerized tomography scans showed a $9.5 \times 8 \mathrm{~cm}$ renal mass in the lower pole of the right kidney. Curative right radical nephrectomy was performed. Pathologic examination showed a clear cell adenocarcinoma. After nephrectomy, the muscle weakness and fasciculations disappeared spontaneously within 2 months. The patient was disease-free for 58 months after right radical nephrectomy. He complained of muscle weakness and fasciculation at the last follow-up again. Physical examination revealed fasciculation in the upper limbs. Abdominal tomography showed a $22 \times 20 \mathrm{~mm}$ solid mass in the lower pole of the left kidney. Kidney-saving surgery was performed and the diagnosis of renal cell carcinoma was confirmed pathologi-
\end{abstract}

cally. Following surgery, fasciculations completely disappeared and muscle weakness diminished within 3 months. Conclusion: This case highlights motor neuron disease as a rare paraneoplastic syndrome in association with renal cell carcinoma and resolution after removal of the tumor.

Copyright $\odot 2008$ S. Karger AG, Basel

\section{Introduction}

Renal cell carcinoma (RCC) is the third most common genitourinary malignancy and its incidence in Middle East countries is less than in Western countries [1]. RCC may lead to a classic triad of symptoms, including flank mass, flank pain and hematuria or to symptoms suggestive of advanced disease, such as weight loss, fever or paraneoplastic syndromes, which occur in approximately $20 \%$ of RCC patients. A wide variety of paraneoplastic syndromes associated with RCC have been described, including anemia, cachexia, fever, hypertension, hypercalcemia, hepatic dysfunction (Stauffer syndrome), amyloidosis, erythrocytosis, enteropathy, and neuromyopathy [2].

Amyotrophic lateral sclerosis (ALS) is a progressive neurodegenerative disorder with a yearly incidence of 1.4-2.7 per 100,000. Approximately $2-5 \%$ of cases of ALS are familial due to mutations of the superoxide dismutase gene [3]. It variably affects motor neurons located in the anterior horns of the spinal cord, the lower cranial motor

\section{KARGER}

Fax +41613061234 E-Mail karger@karger.ch www.karger.com (c) 2008 S. Karger AG, Basel

1011-7571/09/0181-0073\$26.00/0

Accessible online at:

www.karger.com/mpp
H. Mehmet Turk, MD

Department of Clinical Oncology

Faculty of Medicine, Gaziantep University

TR-27310 Gaziantep (Turkey)

Tel. +90 3424720 712, Fax +90 3424720 718, E-Mail turk@gantep.edu.tr 

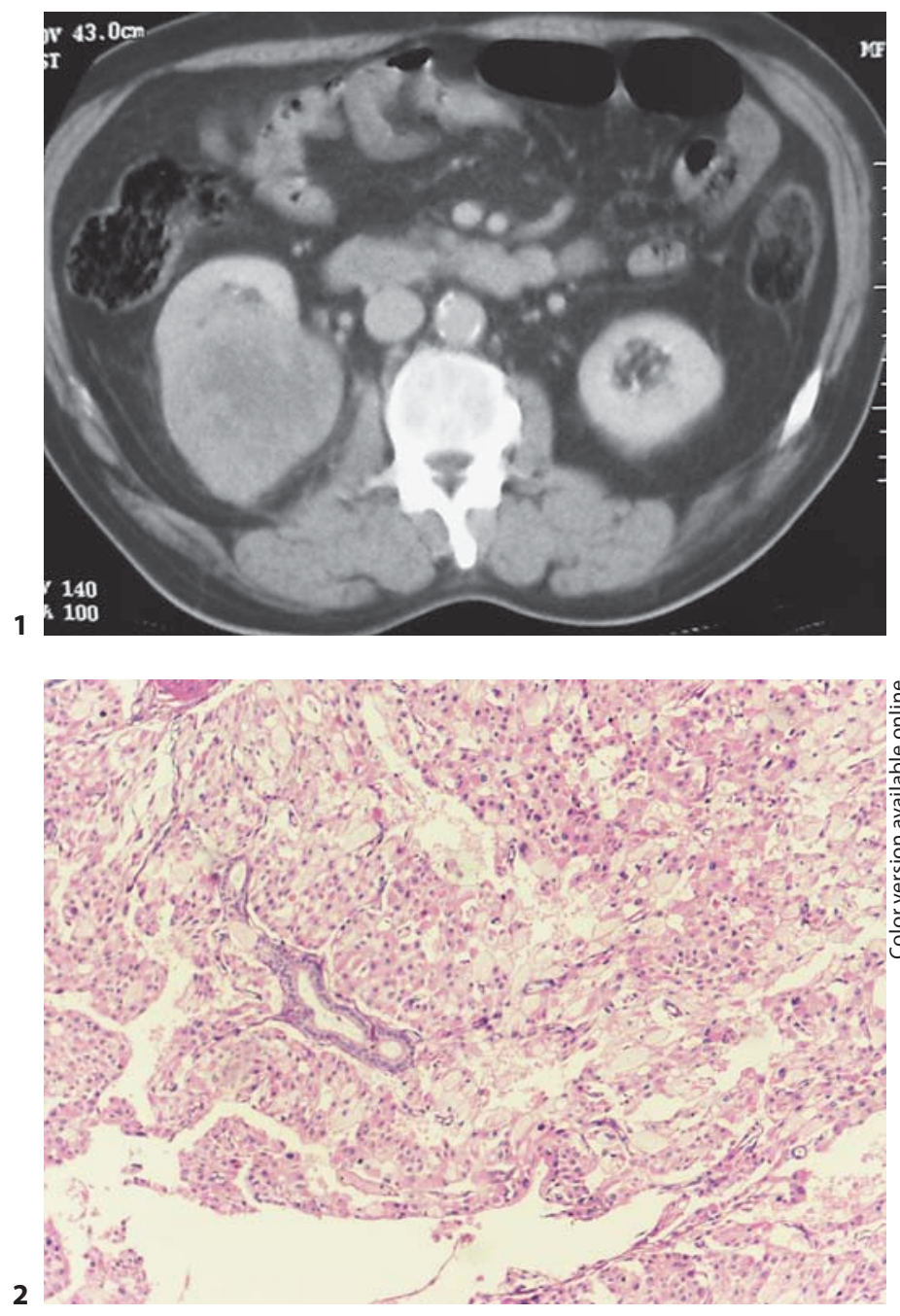

Fig. 1. Abdominal CT scan showing a $9.5 \times 8 \mathrm{~cm}$ heterogeneous renal mass in the lower pole of the right kidney.

Fig. 2. Histological examination of the resected specimen showed RCC of the clear cell type.

nuclei in the medulla, and the frontal cortex. Muscle weakness, atrophy, and fasciculations are a direct consequence of lower motor involvement. Increased muscle stretch reflexes and limb spasticity are due to upper motor neuron involvement. The diagnosis of ALS is established mainly by clinical criteria. Electrodiagnostic examinations such as nerve conduction studies and electromyography may aid to confirm the presence of upper or lower motor neuron involvement. Early cases of motor neuron disease (MND) are very difficult to diagnose whereas advanced cases are diagnosed relatively easily. A positive relationship between MND as a paraneoplastic syndrome and malignant disease has been discussed by many investigators. Although a wide variety of paraneoplastic syndromes associated with RCC have been defined, only a few investigators have reported MND occurring with RCC [4]. We report here a patient with RCC who presented with MND resembling ALS and entered remission after nephrectomy.

\section{Case Report}

A 59-year-old man was admitted because of weakness and fasciculation in the upper extremities lasting for 5 months. He had not been taking any medication and there was no history of drug addiction, toxin exposure or any disease such as diabetes mellitus or neurogenic disorder caused by viral infection and trauma. The neurological examination showed fasciculations that arose spontaneously in the upper limbs and upon triggering in the lower limbs. Electrodiagnostic studies revealed normal nerve conduction with high amplitude motor unit potentials, fibrillation and fasciculation potentials, and incomplete interference pattern in the upper extremities suggesting an active neurogenic disorder. Based on these clinical features and electrodiagnostic findings, the patient was diagnosed with ALS. Whole blood analysis and blood chemistry were all within normal limits. Urine analysis revealed microscopic hematuria. Ultrasonography showed a solid mass (8.5 $\times 7.5 \mathrm{~cm}$ ) in the lower pole of the right kidney. Abdominal computerized tomography CT scans showed a $9.5 \times 8 \mathrm{~cm}$ renal mass in the lower pole of the right kidney. Contrast CT revealed heterogeneous enhancement within the tumor originating in the lower pole of the right kidney and extending to the right hilus while there were no pathologic findings in the left kidney (fig. 1). Curative right radical nephrectomy was performed. Pathologic examination demonstrated a Fuhrman grade I clear cell adenocarcinoma with capsular penetration; Gerota's fascia and distal ureteral and vascular margins were all negative for tumor (fig. 2). Tumor node and metastasis (TNM) staging was T3N0M0. After nephrectomy, the patient received maintenance interferon therapy for 1 year. Muscle weakness and fasciculation disappeared spontaneously within 2 months. The patient was disease-free for 58 months after right radical nephrectomy. However, he complained of muscle weakness and fasciculation at the last follow-up. Physical examination revealed fasciculation in the upper limbs. Abdominal CT showed a $22 \times 20 \mathrm{~mm}$ solid mass in the lower pole of the left kidney and there was no metastatic lesion in the liver. Skeletal scintigraphy was negative for metastasis. Kidney-saving surgery was performed and the diagnosis of RCC was confirmed pathologically. Following surgery, fasciculations completely disappeared and muscle weakness diminished within 3 months. There were no symptoms and signs of relapsed disease for 15 months.

\section{Discussion}

Approximately $15-48 \%$ of RCC cases are diagnosed incidentally during evaluation for an unrelated disorder $[2,5]$. Incidentally discovered RCC tends to be smaller 
and of lower stage. Konnak and Grossman [5] reported that incidental RCC cases tended to be of early stage at presentation, with stage I or II disease in $82 \%$ of incidental versus $30 \%$ of symptomatic cases [5]. Approximately $20 \%$ of RCC patients present with paraneoplastic symptoms. Many symptoms in RCC reflect tumor biology rather than the extent of the tumor. Several studies demonstrated an increased survival rate in incidental RCC [6]. However, recent studies have shown that incidental and symptomatic RCC cases may have similar prognoses [7].

Various paraneoplastic syndromes such as hepatic dysfunction, hypercalcemia, hyperglycemia and hypertension have been reported in association with RCC. Neuromyopathy is one of the rare presentations of paraneoplastic syndromes. MND is another uncommon paraneoplastic syndrome. It has been reported in association with small cell lung cancer, breast cancer, ovarian carcinoma, non-Hodgkin's lymphoma, Hodgkin's disease, and chronic lymphocytic leukemia. ALS may precede or follow the diagnosis of RCC. Our patient was admitted because of weakness and fasciculation in the upper extremities before the diagnosis of RCC. There is no association between ALS and malignancy in epidemiological studies. When physical examination is used for diagnosis, $6.6 \%$ of 1,476 patients with any type of cancer had neuromyopathy. Although neurogenic paraneoplastic syndromes are rare, they frequently precede the diagnosis of cancer and are often encountered when the tumor is small [8]. Although Norris and Engel [9] noted that 10\% of their patients with MND had cancer and proposed that cancer could cause MND, some authors were of the opinion that this association might be coincidental.
Clinical findings of paraneoplastic syndromes may resolve after removal of the neoplastic tissue. Resection of a thymoma can have a dramatic effect on a myasthenic syndrome, and treatment of small cell lung cancer has been noticed to greatly improve Lambert-Eaton syndrome. Cases of hepatic dysfunction, hypercalcemia, hyperglycemia and hypertension resolving after nephrectomy have been reported in patients with RCC $[10,11]$. According to previous reports of patients with MND and RCC, treatment of cancer modified the neurological symptoms of patients [4]. In our case, muscle weakness and fasciculation resolved after nephrectomy. The patient was well until the diagnosis of RCC in the left kidney, when he presented with muscle weakness and fasciculations again. These symptoms diminished for the second time after kidney-saving surgery. Because of the remission in neurological signs after treatment of cancer, we believe that MND was a paraneoplastic syndrome. The same clinical presentations and response to treatment were observed twice, which supports the diagnosis of a paraneoplastic syndrome. It has been reported that the overall survival was not different between patients with ALS and cancer, and patients with ALS only. The cause of death in patients with ALS and cancer was a consequence of the motor neuron syndrome [12].

\section{Conclusion}

This case showed rare paraneoplastic neurological symptoms in association with RCC that resolved after removal of the tumor. Primary resection of the tumor not only improved the quality of life, but also increased the efficacy of immunotherapy.

\section{References}

$>1$ Ghalayini IF, Bani-Hani IH: Detection, staging and clinical implications of renal cell carcinoma. Saudi Med J 2003;24:79-83.

$\checkmark 2$ Kim HL, Belldegrun AS, Freitas DG, Bui MH, Han KR, Dorey FJ, Figlin RA: Paraneoplastic signs and symptoms of renal cell carcinoma: implications for prognosis. J Urol 2003;170:1742-1746.

$\checkmark 3$ Hays AP, Naini A, He CZ, Mitsumoto H, Rowland LP: Sporadic amyotrophic lateral sclerosis and breast cancer: hyaline conglomerate inclusions lead to identification of SOD1 mutation. J Neurol Sci 2006;242:67-69.

4 Evans BK, Fagan C, Amold T, Dropcho EJ, Oh SJ: Paraneoplastic motor neuron disease and renal cell carcinoma: improvement after nephrectomy. Neurology 1990;40:960-962.
5 Konnak JW, Grossman HB: Renal cell carcinoma as an incidental finding. J Urol 1985; 134:1094-1096.

6 Thompson IM, Peek M: Improvement in survival of patients with renal cell carcinoma: the role of the serendipitously detected tumor. J Urol 1988;140:487-490.

7 Mevorach RA, Segal AJ, Tersegno ME, Frank IN: Renal cell carcinoma: incidental diagnosis and natural history: review of 235 cases. Urology 1992;39:519-522.

8 Croft PB, Wilkinson M: The incidence of carcinomatous neuromyopathy in patients with various types of carcinoma. Brain 1965; 88:427-434.
9 Norris FH, Engel WK: Carcinomatous Amyotrophic Lateral Sclerosis. New York, Grune \& Stratton, 1965, pp 24-34.

10 Rosenblum SL: Paraneoplastic syndromes associated with renal cell carcinoma. J S C Med Assoc 1987;83:375-378.

$\checkmark 11$ Steffens MG, de Mulder PH, Mulders PF: Paraneoplastic syndromes in three patients with renal cell carcinoma. Ned Tijdschr Geneeskd 2004;148:487-492.

12 Vigliani MC, Polo P, Chio A, Giometto B, Mazzini L, Schiffer D: Patients with amyotrophic lateral sclerosis and cancer do not differ clinically from patients with sporadic amyotrophic lateral sclerosis. J Neurol 2000; 247:778-782. 